\title{
Dynamics of charged particles in the radio emission region of pulsar magnetosphere ${ }^{\star}$
}

\author{
R. M. C. Thomas and R. T. Gangadhara
}

Indian Institute of Astrophysics, Bangalore 560 034, India

e-mail: [mathew; ganga]@iiap.res.in

Received 7 February 2006 / Accepted 1 March 2007

\section{ABSTRACT}

\begin{abstract}
We consider the classical picture of three dimensional motion of charged particles in pulsar magnetosphere. We adopt a perturbative method to solve the equation of motion, and find the trajectory of particles as they move along the rotating dipolar magnetic field lines. Our aim is to study the influence of rotation on the pulsar radio emission by considering the constrained motion of particles along the open dipolar magnetic field lines. We find that the rotation induces a significant curvature into the particle trajectories. Our model predicts the intensity on leading side dominates over that of trailing side. We expect that if there is any curvature induced radio emission from the region close to the magnetic axis then it must be due to the rotation induced curvature. Our model predicts the radius-to-frequency mapping (RFM) in the conal as well as core emissions.
\end{abstract}

Key words. stars: pulsars: general - acceleration of particles - radiation mechanisms: non-thermal - stars: rotation - radio continuum: stars

\section{Introduction}

The process behind the radio emission in pulsars still evades a concrete understanding. The different types of radiation mechanisms proposed for pulsars are only partially successful in explaining the phenomenon of radio emission. Out of them, the curvature emission emerges as a favorable model which can satisfactorily explain some aspects of pulsar radiation like polarization angle swing. Though it has unresolved issues like the bunching and coherent of emission, it is still believed to be a natural and unavoidable emission process for pulsars (Gil et al. 2004). In this perspective an effort towards understanding the curvature emission process is worthwhile to pursue.

A precise knowledge about the particle motion is required for understanding the curvature emission in pulsars. Over the past three decades almost all the works, which considered the curvature emission in pulsar magnetosphere, assumed that the trajectories of particles are identical to the dipolar field lines along which they are constrained to move. The important effect, which is neglected in this approximation, is the influence of pulsar spin on particle trajectory. However, for an observer in the laboratory frame (inertial frame), the curvature radius of the particle trajectory need not be same as that of the field lines along which they move. Although there are occasional notes about the rotation induced curvature into the particle trajectory (e.g., Hibschman \& Arons 2001), a proper estimation is still lacking.

We restrict our analysis to the region of magnetosphere where the radio emission is prominent. Some limits on the radio emission heights have been estimated by geometrical methods (e.g., Gil \& Kijak 1993; Kijack \& Gil 1997), interstellar scintillation (e.g., Smirnova \& Shishov 1989), and aberration phase shift in pulse profiles (e.g., Blaskiewicz et al. 1991, hereafter BCW91). While discussing the concept of

* Appendices are only available in electronic form at http: //www . aanda . org radius-to-frequency mapping, several authors (Phillips 1992; Kijak \& Gil 1997; Kramer et al. 1997) have set the constraints on the range of radio emission altitudes. Kijak \& Gil (1997) set an upper limit of $10 \% r_{\mathrm{L}}$ for the normal pulsars, where $r_{\mathrm{L}}=P c /(2 \pi)$ is the light cylinder radius, $P$ is the pulsar period and $c$ is the speed of light. Recently, Gangadhara \& Gupta (2001) have analyzed the emission geometry of PSR $0329+54$ and estimated the aberration-retardation $(\mathrm{A} / \mathrm{R})$ phase shift of pulse components by presupposing a nested conal structure for the emission beam. They have found that the radio emission altitude is not constant across the pulse window but it varies in such way that inner cones including the core comes from lower altitude $\left(<1 \% r_{\mathrm{L}}\right)$ compared to the outer ones $\left(\sim 5 \% r_{\mathrm{L}}\right)$. In a subsequent work Gupta \& Gangadhara (2003) have verified it for six more pulsars. Based on these deductions we surmise that an upper limit of $10 \% r_{\mathrm{L}}$ for the radio emission heights of normal pulsars and $20 \% r_{\mathrm{L}}$ for millisecond ones (e.g., Gangadhara 2005a).

We solve the equation of motion and analyze the dynamics of charged particles moving along rotating magnetic field lines. The equation of motion of a particle in the combined electric and magnetic field is given by

$\frac{\mathrm{d} \boldsymbol{p}_{\mathrm{lab}}}{\mathrm{d} t}=q \boldsymbol{E}+\boldsymbol{F}_{\mathrm{B}}$

where $\boldsymbol{p}_{\text {lab }}$ is the momentum of particle in the laboratory frame, $q \boldsymbol{E}$ and $\boldsymbol{F}_{\mathrm{B}}$ are the electric and magnetic parts of the Lorentz force, and $q$ is the particle charge.

The mechanism of initial acceleration of particles in the regions of a few stellar radii has been analyzed by several authors (e.g., Ruderman \& Sutherland 1975; Arons \& Scharlemann 1979; Harding \& Muslimov 1998, hereafter HM98). A simplified picture of initial acceleration, which one can gather from these models, may be summarized as follows. The huge electric fields, which are rotationally generated very close to the neutron star, accelerate the primary particles against gravity to acquire 
very high Lorentz factors of $\approx 10^{5}-10^{7}$. These primary particles in turn emit the pair-producing $\gamma$-ray photons through curvature radiation. These photons in turn produce the secondary electronpositron $\left(\mathrm{e}^{+}, \mathrm{e}^{-}\right)$pair plasma, which further develops into pair formation fronts. By developing a highly sophisticated model for the initial acceleration of charged particles HM98 have shown that secondary particles can screen the accelerating electric fields $\boldsymbol{E}_{\|}=0$ above a distance of about 1-2 stellar radii from the neutron star surface, except in the regions of slot gaps (Muslimov \& Harding 2004). Thus we take that the parallel electric field $\boldsymbol{E}_{\|}=0$ in the radio emission region.

Ruderman \& Sutherland (1975, hereafter RS75) have given an estimate for the Lorentz factor $(\gamma \approx 800)$ of the secondary particles when they come out of the region of huge initial acceleration. Recently, by solving the viewing geometry in dipolar dipolar magnetic field lines, Gangadhara (2004) has deduced Lorentz factor of $(\gamma \approx 300)$ secondary pair plasma. So, we take that the particles, which escape from this region, have Lorentz factor $\gamma \approx 100-1000$. The charged particles, which enter the region of $\boldsymbol{E}_{\|}=0$, experience an acceleration primarily because of curvature in their trajectory, and hence emit curvature radiation.

The multipolar components of pulsar magnetic field are expected to be limited to very low altitudes close to the stellar surface and vanish at higher altitudes leaving a dipolar configuration. Observations indicated that the magnetic field in the radio region is more likely to be dipolar (Xilouris et al. 1996; Kijack \& Gil 1997). An estimate for the azimuthal distortion of magnetic field due to magnetic field line sweep back is given by Dykes \& Harding (2004):

$\delta \phi_{\mathrm{mfsb}} \approx \frac{2}{3} \sin \alpha r_{n}{ }^{3}\left[3 \frac{x y}{r^{2}} \cos \alpha+\left(3 \frac{x^{2}}{r^{2}}-1\right) \sin \alpha\right]^{-1}$,

where $r_{n}=r / r_{\mathrm{L}}, x=r \cos \phi^{\prime} \sin \theta^{\prime}, y=r \sin \phi^{\prime} \sin \theta^{\prime}$ and $z=r \cos \theta^{\prime}$. The angles $\phi^{\prime}$ and $\theta^{\prime}$ are the azimuth and colatitude of the emission spot in a spherical co-ordinate system centered on the rotation axis. The radial distance $r$ is measured from the neutron star center to the emission spot. The value of $\delta \phi_{\mathrm{mfsb}}$ is found to be much less than the $\mathrm{A} / \mathrm{R}$ effects in the region of our interest (Gangadhara 2005b, hereafter G05). Thus we take dipolar magnetic field is represented by

$\boldsymbol{B}=\frac{3(\hat{r} \cdot \boldsymbol{m}) \hat{r}-\boldsymbol{m}}{r^{3}}$

where $\boldsymbol{m}$ is the magnetic dipole moment of the star, and the field lines are governed by

$r=r_{\mathrm{e}} \sin ^{2} \theta$

where $\theta$ is the magnetic colatitude and $r_{\mathrm{e}}$ is the field line constant. Large values of $r_{\mathrm{e}}$ represent the field lines with larger radius of curvature and vice versa.

The drift velocity $v_{\mathrm{cg}}$ of charged particles across the field lines due to curvature and gradient in the magnetic field (Jackson 1975) is given by

$\boldsymbol{v}_{\mathrm{cg}}=\left[\frac{2 v_{\|}^{2}+v_{\perp}^{2}}{2 R \omega_{\mathrm{B}}}\right]\left(\frac{\boldsymbol{R} \times \boldsymbol{B}}{R B}\right)$,

where $v_{\|}$and $v_{\perp}$ are the parallel and perpendicular components of particle velocity with respect to the magnetic field $\boldsymbol{B}, R$ is the radius of curvature of field line, $\omega_{\mathrm{B}}=q B /(m c)$ is the cyclotron frequency, $m$ and $q$ are the mass and charge of particle, respectively. Based on the estimate given in BCW91, we infer $v_{\|} \sim c$ and $v_{\perp} \ll c$. For the typical pulsar parameters we find $v_{\text {cg }} \ll v_{\|}$, and hence we neglect the drift motion.

Machabeli \& Rogava (1994) by considering the particles moving freely along an infinitely long and rigidly rotating straight tube, derived an expression for the particle trajectory. Reiger \& Mannhiem (2000) have also discussed the particle acceleration along the rotating straight magnetic field lines in AGN, by assuming the angular velocity of particles is same as that of AGN. Further, Rogava et al. (2003) have considered the free motion of particles within an arbitrarily curved and infinitely long rotating cylinder.

In our previous work (Thomas \& Gangadhara 2005, hereafter TG05), we considered the two dimensional (2D) geometry and analyzed the dynamics of a charged particle. We approximated the projected field lines on the equatorial plane to be straight lines. However, in the present work we relax this assumption by considering the three dimensional (3D) motion of charged particles in the rotating dipolar field lines. In Sect. 2 we present the solution to the equation of motion, and its solution. We discuss the possible implications of our model in Sect. 3. Concurrently, we also find a numerical solution to the radial position of particle as a function of time in Sect. 3.3, and conclude in Sect. 4. The detailed derivations are provided in Appendices A-E.

\section{Equation of motion and its solution}

We derive and analyse the equation of motion of charged particle moving along the rotating magnetic field lines in the radio emission region. The derivation of equation of motion and its solution are given in detail in Appendices B and C. Using Eq. (C.20), we rewrite the equation of motion Eq. (C.28) as:

$\ddot{r}+\frac{2 \Omega_{\mathrm{m}}^{2} r \dot{r}^{2}+\left(\mathrm{d} \Omega_{\mathrm{m}}^{2} / \mathrm{d} t\right) \dot{r} r^{2}}{c^{2}\left(1-\Omega_{\mathrm{m}}^{2} r^{2} / c^{2}\right)}-\Omega_{\mathrm{m}}^{2} r=0$,

where

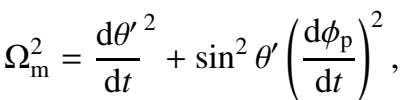

$\dot{r}=\mathrm{d} r / \mathrm{d} t$ and $\ddot{r}=\mathrm{d}^{2} r / \mathrm{d} t^{2}$. Equation (6) is highly nonlinear as it contain the terms in $\dot{r}^{4}$, and the exact analytical solution seems too difficult to achieve. So, we opt for a perturbative method to find solution. Let $\epsilon=r_{\mathrm{L}} / r_{\mathrm{e}}$ be the perturbation parameter. We are limiting the consideration of particle motion to the open field lines for which $r_{\mathrm{e}} \geq 5 r_{\mathrm{L}}$ for the reasons explained in Appendix C. After substituting Eqs. (D.1)-(D.6) into Eq. (6), the coefficients of $\epsilon^{0}$ give the zeroth order equation:

$\ddot{r}_{0}+\frac{2 \Omega_{\mathrm{m} 0}^{2} r_{0} \dot{r}_{0}^{2}}{c^{2}\left(1-\Omega_{\mathrm{m} 0}^{2} r_{0}^{2} / c^{2}\right)}-\Omega_{\mathrm{m} 0}^{2} r_{0}=0$,

and those of $\epsilon^{1}$ give the first order equation:

$$
\begin{gathered}
\ddot{r}_{1}+\frac{4 \Omega_{\mathrm{m} 0}^{2} r_{0} \dot{r}_{0}}{c^{2}\left(1-\Omega_{\mathrm{m} 0}^{2} r_{0}^{2} / c^{2}\right)} \dot{r}_{1}+\left(\frac{2 \Omega_{\mathrm{m} 0}^{2} \dot{r}_{0}^{2}}{c^{2}\left(1-\Omega_{\mathrm{m} 0}^{2} r_{0}^{2} / c^{2}\right)}\right. \\
\left.-\Omega_{\mathrm{m} 0}^{2}+\frac{4 \Omega_{\mathrm{m} 0}^{4} r_{0}^{2} \dot{r}_{0}^{2}}{c^{4}\left(1-\Omega_{\mathrm{m} 0}^{2} r_{0}^{2} / c^{2}\right)^{2}}\right) r_{1}= \\
\Omega_{\mathrm{m} 1}^{2} r_{0}-\frac{2 \Omega_{\mathrm{m} 1}^{2} r_{0} \dot{r}_{0}^{2}+\Omega_{\mathrm{m} 1}^{2} \dot{r}_{0} r_{0}^{2}}{c^{2}\left(1-\Omega_{\mathrm{m} 0}^{2} r_{0}^{2} / c^{2}\right)} \\
-\frac{2 \Omega_{\mathrm{m} 0}^{2} \Omega_{\mathrm{m} 1}^{2} \dot{r}_{0}^{2} r_{0}^{3}}{c^{4}\left(1-\Omega_{\mathrm{m} 0}^{2} r_{0}^{2} / c^{2}\right)^{2}}
\end{gathered}
$$


In our previous work (TG05), we have already found the solution of the zeroth order equation:

$r_{0}=\frac{c}{\Omega_{\mathrm{m} 0}} \operatorname{cn}\left(\lambda-\Omega_{\mathrm{m} 0} \mathrm{t}\right)$,

and to the first order equation, the solution is

$r_{1}=-y_{1} \int \frac{y_{2} \kappa}{w} \mathrm{~d} t+y_{2} \int \frac{y_{1} \kappa}{w} \mathrm{~d} t$,

where the expressions of the parameters $y_{1}, y_{2}, w$ and $\kappa$ are given in Appendix D (see Eq. (D.21)).

\section{Discussion}

Based on our solution to the radial position of the charged particle, we discuss some of the features of particles trajectory and their radius of curvature relevant in the radio emission region of pulsars.

\subsection{Particle trajectory}

Consider a Cartesian coordinate system $(x, y, z)$, whose origin is fixed at the neutron star center and $z$-axis taken along the rotation axis (see Fig. 1). Using Eqs. (D.1), (D.9), (D.20), and (A.1) and (A.4) given in Appendix A, we find the trajectory of particles:

$\boldsymbol{r}=r(t)\left(\sin \theta^{\prime} \cos \phi_{\mathrm{p}} \hat{e}_{\mathrm{x}}+\sin \theta^{\prime} \sin \phi_{\mathrm{p}} \hat{e}_{y}+\cos \theta^{\prime} \hat{e}_{z}\right)$,

where $\left(\hat{e}_{x}, \hat{e}_{y}, \hat{e}_{z}\right)$ are the unit vectors along the $(x, y, z)$ axes, respectively.

In Fig. 2 we have plotted the trajectory of particles moving along the rotating field lines specified by field line constant $r_{\mathrm{e}}=5 r_{\mathrm{L}}$ and magnetic azimuth $\phi= \pm 90^{\circ}$ in the case of an orthogonal rotator. We have plotted the sequential locations of a rotating field line with time step of $0.0055 \mathrm{~s}$ in an inertial frame. The upper-half of the diagram shows the leading side and lower half shows the trailing. The intersection points between the trajectory and field line mark the instantaneous positions of particle during its passage. The continuous solid line, which joins those points, define the trajectory of particle in the laboratory frame (inertial frame). It is clear that they bend towards the direction of rotation (counter-clockwise). Since the field lines on trailing side bend in the opposite direction to rotation, we see an asymmetry in the trajectories between the leading and the trailing sides. In the absence of rotation, trajectories are expected to symmetrically diverge from the magnetic axis as the field lines do, but in the presence of rotation they become asymmetric. It is to be remarked that even for a slight addition of transverse velocity, because of rotation, to the radial velocity $\left(v_{\|} \sim c\right)$ the change that it brings in the radius of curvature is significant. An extreme example would be the case of a particle moving along the rotating magnetic axis. At an altitude of $\sim 0.1 r_{\mathrm{L}}$ the value of radius of curvature $\rho=\infty$ if no rotation, but with rotation velocity $(<0.1 c)$ it would be $\rho \approx r_{\mathrm{L}} /(2 \sin \alpha)$.

In Fig. 3, the trajectories of particles moving along the magnetic axis $\left(\theta=0^{\circ}, \theta^{\prime}=\alpha\right.$ and $\left.\phi=0^{\circ}\right)$ are plotted for the different cases of inclination angle $\alpha$. We infer, the trajectories are curved towards the direction of rotation, and the radius of curvature of the trajectory increases with the decreasing $\alpha$, as the rotation effects are more significant in the case of orthogonal rotators. It demonstrates the influence of rotation on the trajectory of particles in laboratory frame. Even though the magnetic axis is straight, the trajectory of particles are not as they travel in time

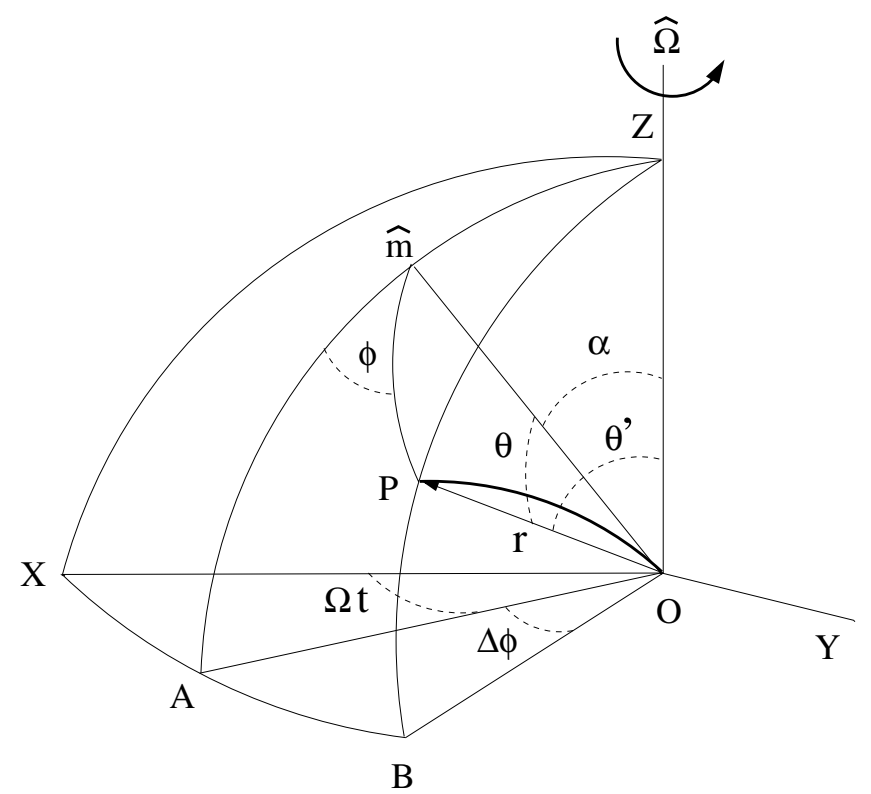

Fig. 1. The geometry showing the motion of a particle along a rotating magnetic field line (thick curve PO) in the laboratory frame. The phase difference $\Delta \phi$ is an angle between the projected magnetic axis $\mathrm{AO}$ and the projected radial vector $\mathrm{OB}$ on the $X Y$-plane. The angle $\angle X \mathrm{OA}=$ $\Omega t$ is the phase of projected magnetic axis with respect to $X$-axis of a fixed Cartesian coordinate system- $X Y Z$ whose $Z$-axis is parallel to the rotation axis $\hat{\Omega}$.

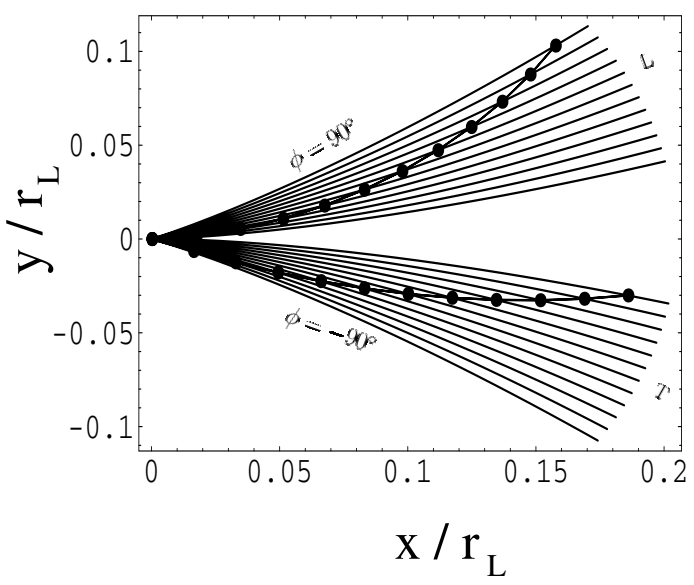

Fig. 2. A diagram showing how the rotating field lines influence the particle trajectory on the leading (L) and trailing (T) sides of the magnetic axis. The several lines on the upper-half of the diagram represent the sequential rotated positions of a single field line with a time step of $0.0055 \mathrm{~s}$. Similarly, those on the lower-half the figure mark the sequential location of field lines on trailing side. The intersections (marked with bullets) between the field line and trajectory specify the instantaneous location of particle in the observer frame (inertial frame). These trajectories are computed by considering the field lines specified by $r_{\mathrm{e}}=5 r_{\mathrm{L}}$ and $\phi= \pm 90^{\circ}$ on the equatorial plane of an orthogonal rotator having spin period of $1 \mathrm{~s}$.

from slowly rotating region (polar cap) to fast rotating region (light cylinder). We note that the relativistic particles moving in such curved trajectories emit the curvature radiation.

To further illustrate the influence of rotation on particle trajectories, we have plotted them on the equatorial plane (see Fig. 4) of a pulsar with $\alpha=90^{\circ}$ and $P=1 \mathrm{~s}$. We have selected two open field lines $r_{\mathrm{e}}=5 r_{\mathrm{L}}$ : one on the leading side $\left(\phi=90^{\circ}\right)$ and other on the trailing side $\left(\phi=-90^{\circ}\right)$. For 


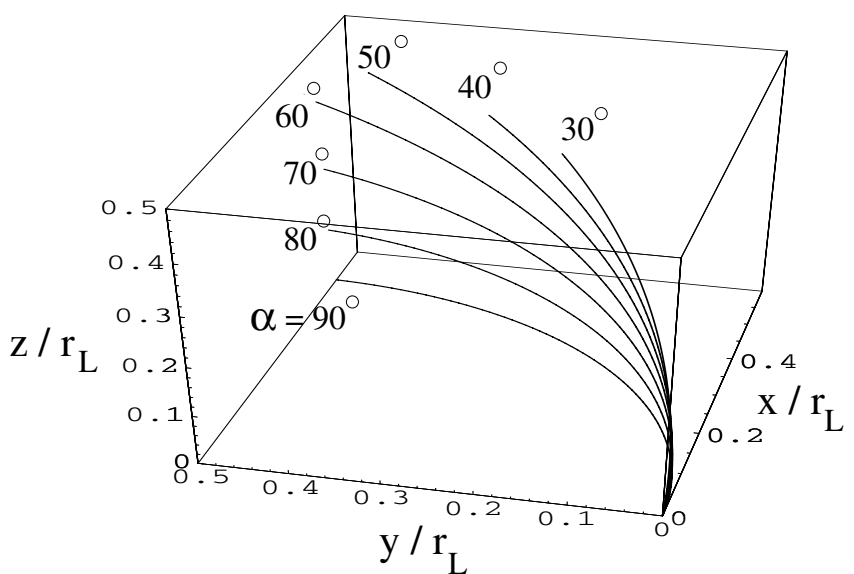

Fig. 3. The trajectories of particle moving along the magnetic axis at different inclination angle $\alpha$. The azimuthal distortion due to the magnetic field sweep back is neglected in this plot. Used pulser period $P=1 \mathrm{~s}$ and $\phi=0^{\circ}$. The curve labeled with $\alpha=90^{\circ}$ lies in the $x y$-plane, and all other lie above it.

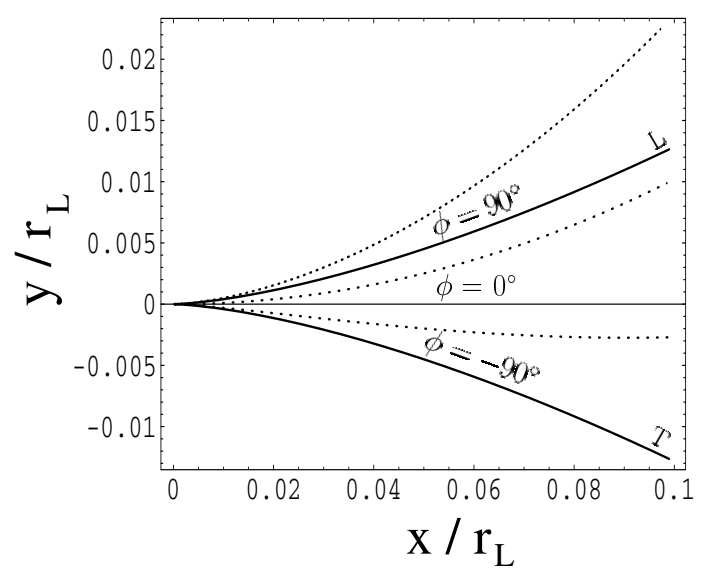

Fig. 4. The projected trajectories of particles moving along the field lines with $\phi=90^{\circ}$ on leading side, and $\phi=-90^{\circ}$ on trailing side, on the equatorial plane. The solid lines represent the magnetic field lines while the dotted ones mark the particle trajectories. Chosen $\alpha=90^{\circ}$ and $P=1 \mathrm{~s}$.

reference we have also plotted the trajectory of particle moving along the magnetic axis $\left(\phi=0^{\circ}\right)$. Based on Figs. 2-4 we make the following observations in the laboratory frame: (1) the trajectories are deflected towards the direction of rotation with respect to the field lines, and the magnitude of deflection increases with the radial distance from the rotation axis; (2) the magnitude of deflection on leading side is larger than the trailing side, i.e., an asymmetric deflection seen. This is because of the fact that the dipolar field lines bend toward the direction of rotation on leading side whereas on trailing side they bend in the opposite directions. Hence we expect the rotation induced curvature emissions on leading side to dominate over the trailing side.

\subsection{Radius of curvature of particle trajectory}

In a corotating frame particles are expected to move with relativistic velocities $\boldsymbol{v}_{\|} \sim c$ along the field lines with Lorentz factor $\gamma \sim 100-1000$. But for an observer in the Laboratory frame particles move relativistically along the field lines as well as rotating with the velocity $\boldsymbol{v}_{\text {rot }}=\Omega \times \boldsymbol{r}$. The emitted radiation will be

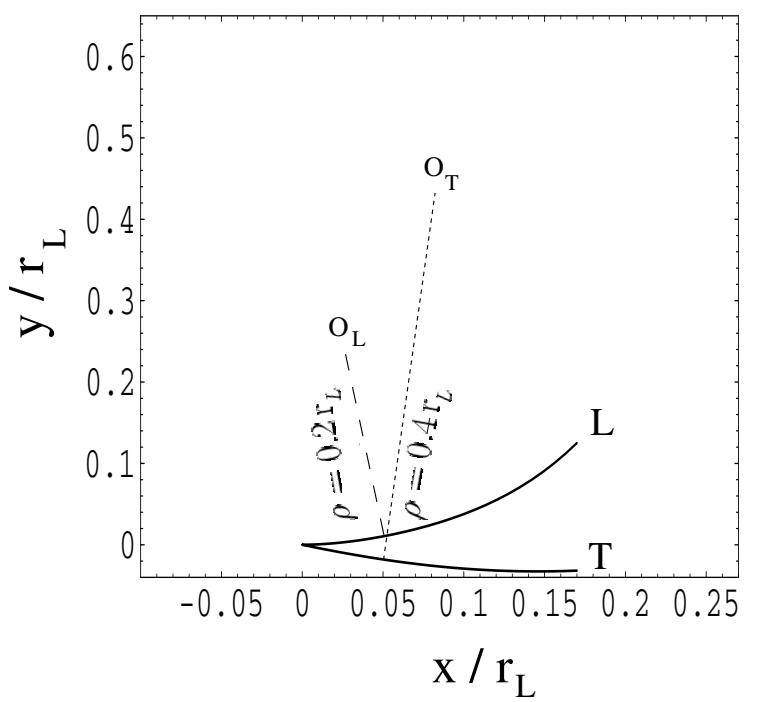

Fig. 5. The curvature radii for the trajectories $\mathrm{L}$ and $\mathrm{T}$ on the leading and trailing sides (see Fig. 2). At $x=0.05 r_{\mathrm{L}}$ the computed values of curvature radii are $\rho=0.2 r_{\mathrm{L}}$ and $0.4 r_{\mathrm{L}}$ with local centers at $O_{\mathrm{L}}$ and $O_{\mathrm{T}}$ for the leading and trailing side trajectories, respectively.

beamed in the direction of total velocity: $\boldsymbol{v}_{\text {lab }}=\boldsymbol{v}_{\|}+\boldsymbol{v}_{\text {rot }}$. Since rotation velocity is a function of radial distance $r$ from the rotation axis, it increases with altitude. It is quite small at the polar cap and approaches the speed of light $(c)$ near the light cylinder. In the radio emission zone ( $r \sim 200$ to $2000 \mathrm{~km}$ ) of normal pulsars, the rotation velocity is about 2 to 10 per cent of $c$ for the spin periods of 1 to $0.1 \mathrm{~s}$. Further, in millisecond pulsars it is still larger. Therefore, with an increasing $r$ the trajectory of particles, which corotate with the magnetic field lines, become more and more curved towards the direction of rotation. Even if we take a straight field line, the trajectory of particle becomes curved for an observer in the laboratory frame, as the particle gets differentially shifted towards the direction of rotation. This introduces a real physical curvature into the trajectory of particle in the laboratory frame. Though at any particular instant an inertial observer can see only a small segment of the trajectory, as the time progresses he will be able to see the full trajectory due to pulsar rotation.

The curvature radius of particle trajectory can be estimated using the following expression

$\rho=\frac{|\boldsymbol{v}|^{3}}{|\boldsymbol{v} \times \boldsymbol{a}|}$,

where $\boldsymbol{v}$ and $\boldsymbol{a}$ are the particle velocity and acceleration, respectively. In Fig. 5, we have plotted the trajectory of particles and their curvature radii on leading and trailing sides as fractions of light cylinder radius $r_{\mathrm{L}}$. At $x=0.05 r_{\mathrm{L}}$, we have marked their local center of curvature and curvature radii. We find curvature radius on leading side $\left(0.2 r_{\mathrm{L}}\right)$ becomes smaller than that on trailing side $\left(0.4 r_{\mathrm{L}}\right)$. The "projected acceleration" can be given as $a_{\text {proj }}=v^{2} / \rho \approx c^{2} / \rho$, where $v$ is the instantaneous velocity of particle and $\rho$ the radius of curvature, which is influenced by the rotation. Hence the emissions on leading side are expected to be stronger than the trailing side, in agreement with the results anticipated by Blaskiewicz et al. (1991) \& Peyman \& Gangadhara (2002).

By considering the motion of particles along the field lines, which lie in the meridional plane defined by $(\hat{\Omega}, \hat{m})$, we have plotted the curvature radius as a function of $r / r_{\mathrm{L}}$ in Fig. 6. 

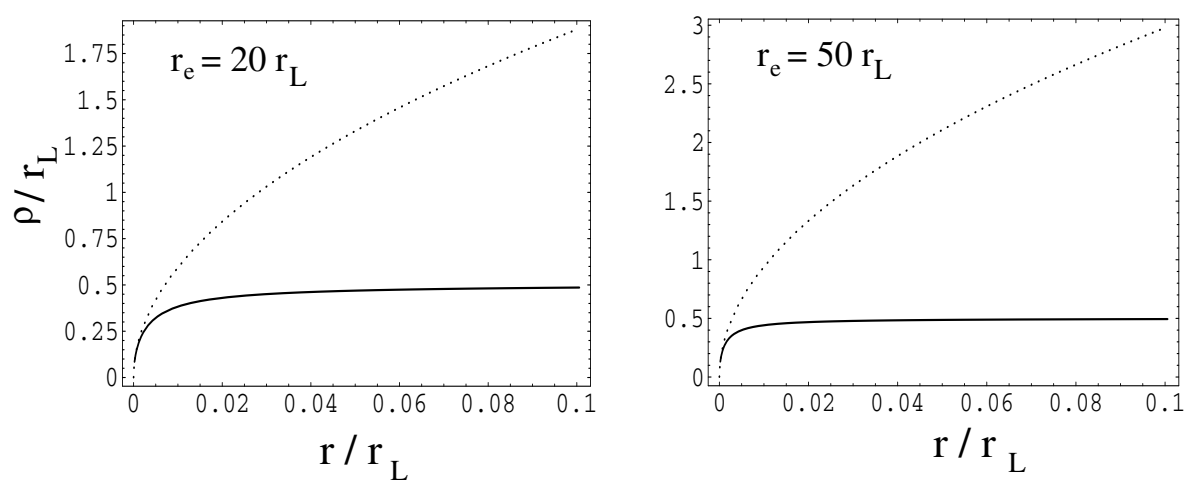

Fig. 6. The curvature radius of particle trajectory at different $r_{\mathrm{e}}$ of the field lines which lie in the meridional plane $\left(\phi=0^{\circ}\right)$. In each panel, the dotted curve represent the stationary case while the continuous curve represent the rotating. Chosen $\alpha=90^{\circ}$ and $P=1 \mathrm{~s}$.
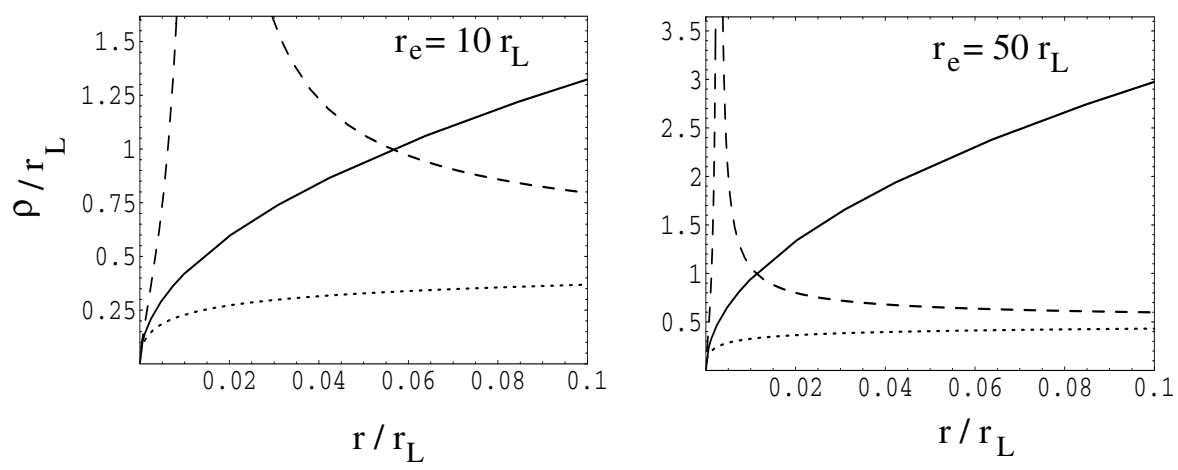

Fig. 7. The curvature radius of particle trajectory at different $r_{\mathrm{e}}$ of the field lines which lie in the leading $\left(\phi=-90^{\circ}\right)$ and trailing sides $\left(\phi=90^{\circ}\right)$. In each panel, the continuous curve represent the stationary case $\left(\phi= \pm 90^{\circ}\right)$, while in the rotating case the dashed line curve represent the trailing $\left(\phi=-90^{\circ}\right)$ side and the dotted one the leading side $\left(\phi=90^{\circ}\right)$. Chosen $\alpha=90^{\circ}$ and $P=1 \mathrm{~s}$.

It shows that when the rotation is taken into account the curvature radius of particle trajectory becomes far lesser than the intrinsic curvature of the field lines. The value of $\rho$ approaches the limiting value $\approx r_{\mathrm{L}} /(2 \sin \alpha)$ at higher altitudes for the field lines closer to the magnetic axis, as the rotation picks up and induces curvature into the trajectory. The curves are roughly flat at higher altitudes $\left(r / r_{\mathrm{L}}>0.01\right)$ because of the fact that the intrinsic radius of curvature of field lines increases with altitude but the rotation induced radius of curvature decreases as the rotation picks up at larger altitude. Though the inherent curvature radii of these field lines are much above $r_{\mathrm{L}} /(2 \sin \alpha)$, it is the rotation which brings radius of curvature of particle trajectory to this limiting value of $\sim r_{\mathrm{L}} / 2$. It is also evident from the approximate expression of $\rho_{\mathrm{m}}$ (see below). For Fig. 7, we considered the field lines which lie outside the meridional plane $\left(\phi= \pm 90^{\circ}\right)$. It shows due to rotation the curvature radius on the leading side becomes smaller than that on the trailing side. Since the field lines having large (say $r_{\mathrm{e}}>100$ ) are almost straight, the difference in curvature radii between the trailing and leading sides is minimal at higher altitudes $\left(r / r_{\mathrm{L}}>0.01\right)$.

In most of the related works the curvature of particle trajectory has been assumed to be same as that of the intrinsic curvature radius of the dipolar field lines along which they are constrained to move. However, our model shows the assumption of identifying the curvature radius of particle trajectory with the curvature radius of field lines can not be extended to the radio emission region.
Though it is difficult to find a simple expression for the curvature radius to illustrate the effects of rotation based on Eq. (13), we can find an approximate expression

$\rho_{\mathrm{m}} \approx \frac{v^{2}}{|\boldsymbol{a}|}$

for a particle moving on a field line very close to the magnetic axis. The parameter $\rho_{\mathrm{m}}$ designates the approximate expression for the curvature radius when the rotation is taken into account. Consider the approximate forms of Eqs. (A.6) and (A.7):

$\frac{\mathrm{d} \theta^{\prime}}{\mathrm{d} t} \approx \cos \phi \frac{\mathrm{d} \theta}{\mathrm{d} t} \approx \frac{\cos \phi}{2} \frac{\dot{r}}{\sqrt{r r_{\mathrm{e}}}}$,

and

$\sin \theta^{\prime} \approx \sin \alpha$.

The term $\mathrm{d} \theta^{\prime} / \mathrm{d} t \approx 0$ as $r_{\mathrm{e}} \gg r$ for the field lines which are close to the magnetic axis. Next by taking $\boldsymbol{v}=c \boldsymbol{\beta}, \boldsymbol{a}=\mathrm{d} \boldsymbol{v} / \mathrm{d} t$ and $\mathrm{d} \phi_{\mathrm{p}} / \mathrm{d} t \approx \Omega$ at $\phi \approx 0^{\circ}$, we obtain

$\rho_{\mathrm{m}} \approx \frac{c}{\Omega_{\mathrm{m} 0}}\left[4+\left(\csc ^{2} \alpha-1\right) \sin ^{2}\left(\Omega_{\mathrm{m} 0} t\right)\right]^{-1 / 2}$.

For $\alpha=90^{\circ}$ we find $\rho_{\mathrm{m}} \approx r_{\mathrm{L}} / 2$, which is in agreement with our earlier estimate in the 2D case (TG05).

A general expression relating the radius of curvature of trajectory of particles moving on a rotating field line:

$\rho_{\text {rot }}=\rho_{\text {dipole }}\left(1+\frac{v_{\text {rot }}^{2}}{v_{0}^{2}}\right) /\left(\sqrt{1+\frac{a_{\text {rot }}^{2}}{a_{0}^{2}}}\right)$ 

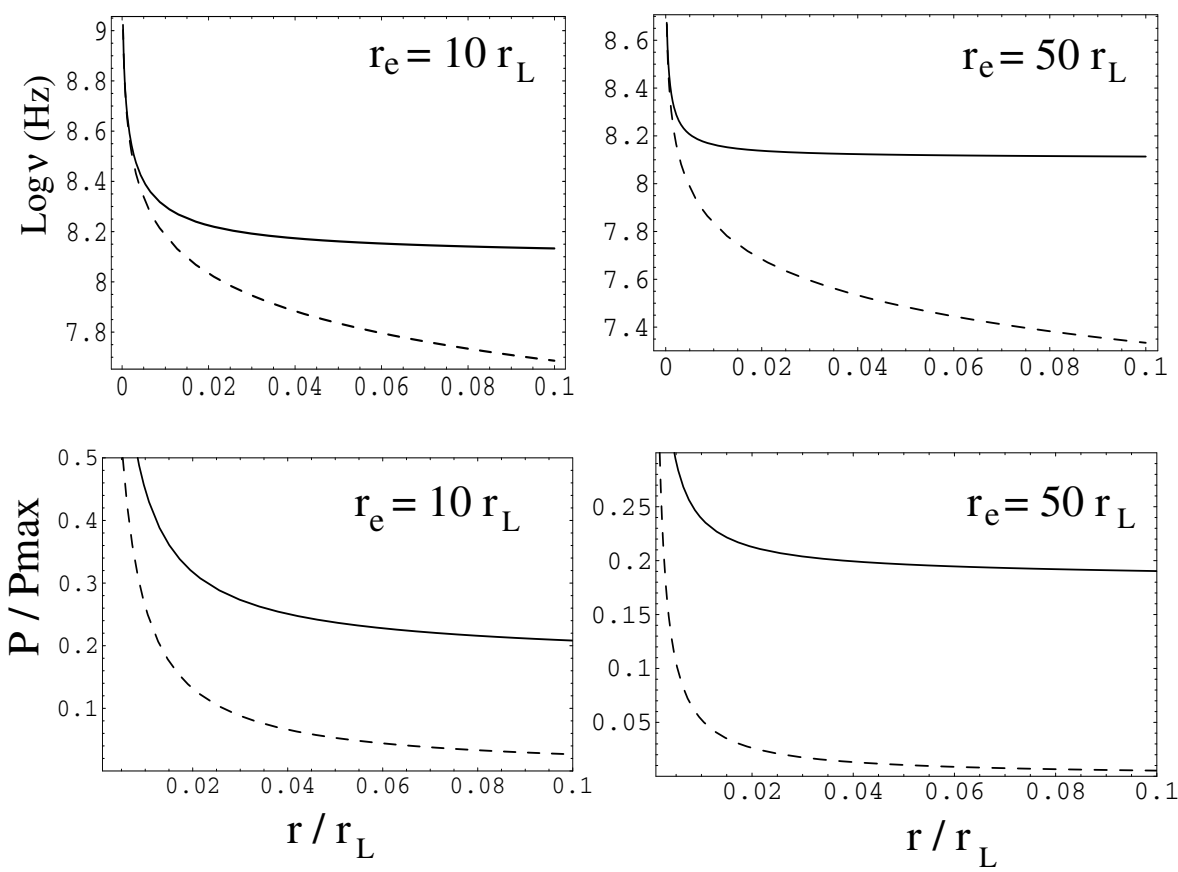

Fig. 8. The characteristic frequency $(v)$ and power $(P)$ emitted by a charged particle moving along the field lines with different $r_{\mathrm{e}}$, in both rotating and non-rotating cases, are plotted as functions of $r / r_{\mathrm{L}}$. In each panel, the rotating case is plotted with a solid line while the static case with a dashed. The power emitted is normalized with $P_{\max }=6.5 \times 10^{-25} \mathrm{erg} / \mathrm{s}$. Chosen $\alpha=90^{\circ}, \gamma=350, P=1 \mathrm{~s}$ and $\phi=0^{\circ}$.

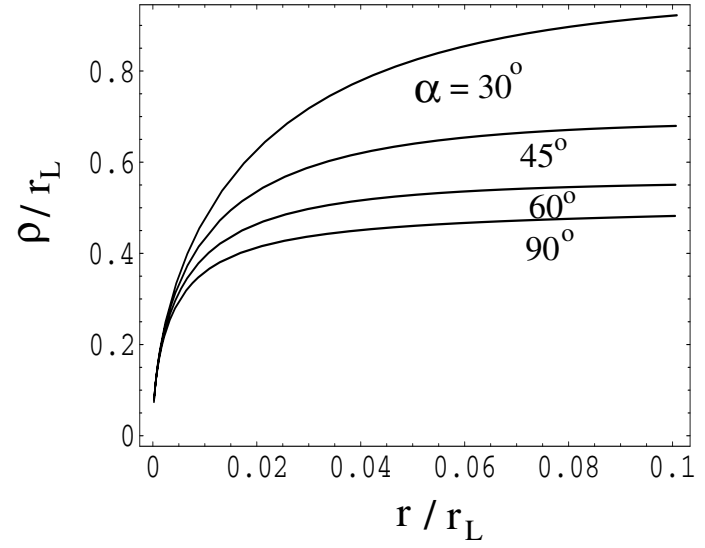

Fig. 9. The curvature radius vs. $r / r_{\mathrm{L}}$ at different angles of inclination $\alpha$ for particles following the field line with $r_{\mathrm{e}}=15 r_{\mathrm{L}}$. Chosen $\phi=0^{\circ}$ and $P=1 \mathrm{~s}$.

where $\rho_{\text {dipole }}$ is the radius of curvature of non-rotating dipole filed line, and the details are given in Appendix E. The expression for $\rho_{\text {dipole }}$ is given by Eq. (4) in G04. For the altitude very close to the stellar surface both the terms $v_{\text {rot }}$ and $a_{\text {rot }}$ become negligible in comparison to $v_{0}$ and $a_{0}$. But for higher altitudes $v_{\text {rot }}$ and $a_{\text {rot }}$ become significant, and hence $\rho_{\text {rot }}$ differs from $\rho_{\text {dipole }}$ as indicated by Figs. 6 and 7.

Gupta \& Gangadhara (2003) by analyzing the profiles of a set of six pulsars indicated that the conal emissions are coming from the field lines whose foot points on the polar cap are within the range of approximately 0.2 to $0.7 S_{\mathrm{L}}$. The polar cap radius $S_{\mathrm{L}}=\sqrt{r_{\mathrm{S}}^{3} / r_{\mathrm{L}}}$, where $r_{\mathrm{s}} \approx 10 \mathrm{~km}$ is the neutron star radius. For typical classical pulsars the value of $S_{\mathrm{L}}$ lies in the range of 100 to $200 \mathrm{~m}$, and the conal rings may be separated by 10 to $20 \mathrm{~m}$ on the polar cap (Gupta \& Gangadhara 2003). So, in the picture of nested conal structure, the central component (core)

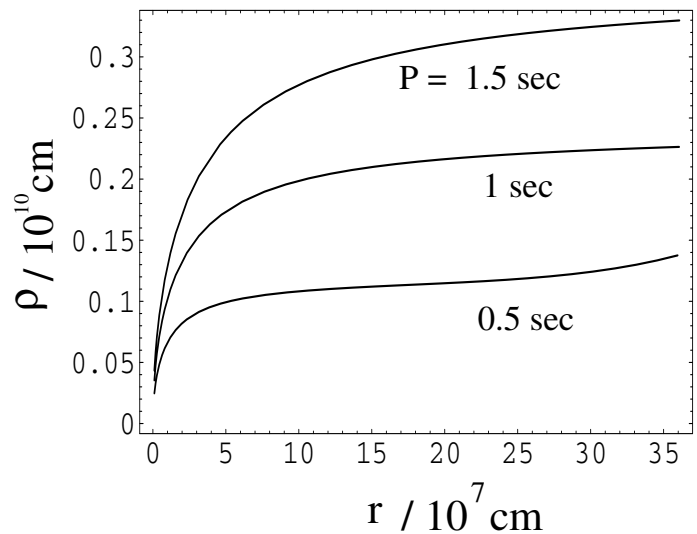

Fig. 10. The curvature radius vs. $r / r_{\mathrm{L}}$ at different pulsar rotation periods $P$ based on Eq. (13). Chosen $\alpha=90^{\circ}, \phi=0^{\circ}$ and $r_{\mathrm{e}}=15$.

is expected to occupy a region with radius of about 15 to $20 \mathrm{~m}$ around the magnetic axis. We find the constant $r_{\mathrm{e}}$ of field lines associated with different emission components approximately lie in the range of 5 to $70 r_{\mathrm{L}}$ for the conal components and greater than $70 r_{\mathrm{L}}$ for the core.

The characteristic frequency $(v)$ and the power $(P)$ emitted by a particle due to curvature radiation (RS75) are given by

$v=\frac{3}{4 \pi} \gamma^{3} \frac{c}{\rho}$

and

$P=\frac{2}{3} \frac{q^{2}}{c} \gamma^{4}\left(\frac{c}{\rho}\right)^{2}$

In Fig. 8 , we plotted $v$ and $P$ in both the rotating (continuous lines) and the non-rotating (dashed lines) cases. When the rotation is considered we find significantly enhanced emission 

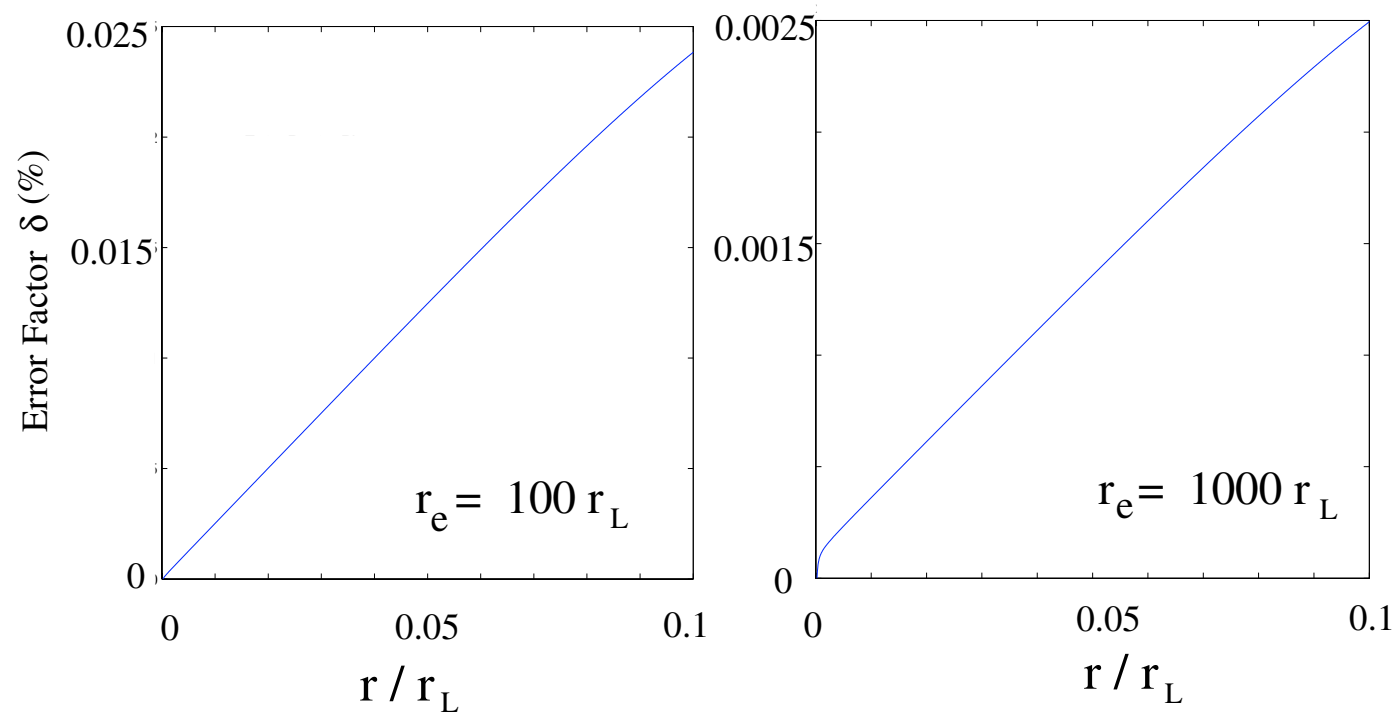

Fig. 11. The analytical solution $(r)$ up to the first order in $\epsilon$ of the radial equation of motion is compared with the corresponding precise numerical solution $\left(r_{\mathrm{n}}\right)$ for different field line constant $r_{\mathrm{e}}$. The error factor $\delta(\%)=\left(1-r / r_{\mathrm{n}}\right) \times 100$ is plotted with respect to the fractional radial distance $r / r_{\mathrm{L}}$. Chosen $\alpha=90^{\circ}$ and pulsar period $P=1 \mathrm{~s}$.

compared to the non-rotating case. In the case of emissions from the field lines close to the magnetic axis the disparity in the values of frequency and power emitted between the rotating and non-rotating cases is huge. This again emphasizes the importance of including the rotation on pulsar radio emission.

Many normal pulsars have the average pulse profiles with very prominent core component. If the core emission is believed to occur from the field lines, which are close to the magnetic axis then it becomes difficult to account for the strong core due to the intrinsic curvature emission, which do not invoke rotation. This is because the field lines are almost straight $\left(\rho_{i} \sim \infty\right)$, and hence the intrinsic curvature emission is expected to become inefficient.

Other aspect of $\rho_{\mathrm{m}}$ to be noted is its dependence on the inclination angle $\alpha$ and the pulsar period $P$, and it can be easily understood from Eq. (15). We find that $\rho_{\mathrm{m}}$ decreases with the increase of $\alpha$ (see, Fig. 9): minimum for orthogonal rotators but maximum in nearly aligned ones. But $\rho_{\mathrm{m}}$ increases with the increase of pulsar period $P$ (see, Fig. 10): larger for classical pulsars than in millisecond pulsars. However, the $\rho_{i}$ does not have any such variations. In literature, $\rho_{\mathrm{m}}$ of the particle trajectory has been often taken to be of the order of $10^{9} \mathrm{~cm}$ (e.g., Zhang \& Cheng 1995; Harko \& Cheng 2002; Gil et al. 2004) or assumed to be same as $\rho_{\mathrm{i}}$. We emphasis that by making such an approximation we miss out an important physics behind the influence of rotation.

Asymmetry in the curvature radius of particle trajectories on leading and trailing sides with respect to the magnetic axis is quite evident from Fig. 5. The leading side trajectories are found to have smaller curvature radius than those on the trailing side. These differences are further reflected in the generation of asymmetric profiles in such way that the leading side components become more intense compared to those on the trailing side in a pulse profile. This feature was first remarked in BCW91 that out of the 88 cone-dominated pulsars 53 pulsars have the leading component stronger while only 24 pulsars have the trailing component stronger. One can intuitively understand this as a consequence of the fact that rotation increases the curvature of particle trajectory on the leading side but reduces on the trailing side.
Thus owing to rotation, particle trajectory becomes more curved on the leading side but stretched out on the trailing side, as indicated by Fig. 2. From Eq. (14) we observe though $|\boldsymbol{v}|$ of particle remain much closer to the speed of light $c$, the acceleration $|\boldsymbol{a}|$ will be much less on trailing side than on leading as indicated by Fig. 7. Recently, Peyman \& Gangadhara (2002) have analyzed the profiles of 24 pulsars, and reported that in 19 pulsars the leading side components are broader than their trailing side counter parts. It is in clear agreement with Fig. 4 that due to rotation the relativistically beamed emissions on the leading side are spread out in angle compared to the trailing side.

The characteristic frequency of the curvature emission is inversely proportional to the curvature radius of particle trajectory (RS75). Hence from Figs. 6 and 7 we expect that the high frequency radiation arises from lower altitudes than the lower frequency one. This implies the existence of radius-to-frequency (RFM) mapping in the case of emission from field lines close to magnetic axis. If core emission occurs from these field lines then we expect that the core emission heights also follow the RFM. Since the curvature radius increases steeply at lower altitudes $\left(r / r_{\mathrm{L}} \leq 0.01\right)$, most of the high frequency radiation is expected to be generated over a narrow range of altitude. But at higher altitudes curvature radius increases less steadily with respect to $r$. Hence the lower frequency emission region is found to be distributed over much a larger range of altitude than the high frequency radiation. It is in clear agreement with the conclusion drawn by Hoensbroech \& Xilouries (1997) that the RFM is evident at cm-wavelengths but saturates progressively with the increase of frequency.

Since the millisecond pulsar rotates much faster than the normal ones it is natural to expect that the effects of rotation to be more prominent in them. An easily detectable and a direct prominent effect of rotation in millisecond pulsars is the larger aberration-retardation effect in comparison to normal pulsars. For example, one can look at our recent work on a nearby and strong millisecond pulsar PSR J0437-4715 (Gangadhara and Thomas), where we find that the aberration-retardation phase shift is as high as $23^{\circ}$, a value much larger than the one ever detected in normal pulsars. Further example is the work of 
Johnston \& Weisberg (2006) on PSR J1015-5719, which has a spin period of $139.9 \mathrm{~ms}$. They have detected the aberrationretardation phase shifts, which are much larger than the normal pulsars (Gangadhara \& Gupta 2001; Gupta \& Gangadhara 2003).

\subsection{Numerical solution}

To estimate the accuracy of our analytical solution $r=r_{0}+r_{1}$, we numerically solved Eq. (6) using Eq. (7). Equation (6) was split into two first order equations given by

$p=\dot{r}$

and $\dot{p}=f(r, \dot{r})$,

where $p$ is a dummy variable and

$f(r, \dot{r})=-\frac{2 \Omega_{\mathrm{m}}^{2} r \dot{r}^{2}+\left(\mathrm{d} \Omega_{\mathrm{m}}^{2} / \mathrm{d} t\right) \dot{r} r^{2}}{c^{2}\left(1-\Omega_{\mathrm{m}}^{2} r^{2} / c^{2}\right)}+\Omega_{\mathrm{m}}^{2} r$.

By choosing $\alpha=90^{\circ}$ and $\phi=0^{\circ}$, we numerically solved the above system of coupled first order equations using Matlab ODE-solvers. They have been set as a bench mark for testing the accuracy of the perturbative solution. To compare the numerical solution $\left(r_{\mathrm{n}}\right)$ with the analytical solution $(r)$, we have plotted the error factor $\delta(\%)=\left(1-r / r_{\mathrm{n}}\right) \times 100$ with respect to the fractional radial distance $\left(r / r_{\mathrm{L}}\right)$ in Fig. 11. It shows error factor $\delta(\%)$ is less than $0.025 \%$ in the radio emission region.

\section{Conclusion}

By taking into account of pulsar rotation, we have developed a 3D model for the motion of charged particles in the radio emission region of pulsar magnetosphere. We have solved the equation of motion by perturbative method and obtained the trajectory of particles. For an observer in the laboratory frame, trajectories are found to have clear deviations from the geometry of dipolar field lines. Due to rotation, the trajectories become more curved on the leading side of magnetic axis than on the trailing side. Particles emit curvature radiation due to the curvature introduced into their trajectory by both the intrinsic curvature of dipolar field lines and the rotation. The emissions on the leading side are expected to dominate over that on the trailing side. We expect that the emissions close to the magnetic axis (core) includes a major contribution from the rotation induced curvature emission. Our model predicts radius-to-frequency mapping for both the core and the conal emissions.

Acknowledgements. We thank Vikram S. Chettiar for his valuable contributions in discussions and numerical computations required for this work.

\section{References}

Abramowitz, M., \& Stegun, I. A. 1972, A Hand Book of Mathematical Functions (NY: Dover Publications, Inc.)

Arons, J., \& Scharlemann, E. T. 1979, ApJ, 231, 854

Blaskiewicz, M., Cordes, J. M., \& Wasserman, I. 1991, ApJ, 370, 643 (BCW91) Chedia, O. V., Kahniashvili, T. A., Machabeli, G. Z., \& Nanobashvili, I. S. 1996, Ap\&SS, 239, 57

Dyks, J., \& Harding, A. K. 2004, ApJ, 614, 869

Endean, V. G. 1974, ApJ, 187, 359

Gangadhara, R. T. 1996, A\&A, 314, 853

Gangadhara, R. T. 2004, ApJ, 609, 335 (G04)

Gangadhara, R. T. 2005a, [arXiv: astro-ph/0411161] (v2)

Gangadhara, R. T. 2005b, ApJ, 628, 923 (G05)

Gangadhara, R. T., \& Gupta, Y. 2001, ApJ, 555, 31

Gil, J. A., \& Kijak, J. 1993 A\&A, 273, 563

Gil, J. A., Lyubarsky, Y., \& Melikidze, G. I. 2004, ApJ, 600, 872

Gupta, Y., \& Gangadhara, R. T. 2003, ApJ, 584, 418

Harding, A. K., \& Muslimov, A. G., 1998, ApJ, 508, 328 (HM98)

Harko, T., \& Cheng, K. S. 2002, MNRAS, 335,99

Hibschman, J. A., \& Arons, J. 2001, ApJ, 546, 382

Hoensbroech, von, A., \& Xilouris, K. M. 1997, A\&A, 324, 981

Jackson, J. D. 1975, Classical Electrodynamics (NY: Wiley)

Kijak, J., \& Gil, J. 1997, MNRAS, 288, 631

Kramer, M., Xilouris, K. M., Jessner, A., et al. 1997, A\&A, 32, 846

Machabeli, G. Z., \& Rogava, A. D. 1994, Phys. Rev. A, 50, 98 (MR94)

Mestel, L. 1973, Ap\&SS, 24, 289

Muslimov, A. G., \& Harding, A. K. 2004, ApJ, 606, 1143

Peyman, A., \& Gangadhara, R. T. 2002, ApJ, 566, 365

Phillips, J. A. 1992, ApJ, 385, 282

Rieger, F. M., \& Mannheim, K. 2000, A\&A, 353, 473

Rogava, A. D., Dalakishvili, G., \& Osmanov, Z. 2003, Gen. Rel. Grav., 35, 1133

Ruderman, M., \& Sutherland, P. 1975, ApJ, 196, 51 (RS75)

Smirnova, T. V., \& Shyshov, V. I. 1989, PAZh, 15, 443

Thomas, R. M. C., \& Gangadhara, R. T. 2005, A\&A, 437, 537 (TG05)

Xilouris, K. M., Kramer, M., Jessner, A., Wielebinski, R., Timofeev, M. 1996, A\&A, 309, 481

Zhang, J. L., \& Cheng, K. S. 1995, AcASn., 36, 437

Zwillinger, D. 1989, Handbook of Differential Equations (Academic Press Inc.) 
R. M. C. Thomas and R. T. Gangadhara: Radio emission in pulsars, Online Material $p 1$

\section{Online Material}




\section{Appendix A: The small angle approximations}

For analytical simplicity we introduce the following small angle relations:

$$
\begin{aligned}
\theta^{\prime} & =\theta_{d}+\alpha \quad \text { and } \\
\cos \theta^{\prime} & =\cos \left(\theta_{d}+\alpha\right) \\
& =\cos \alpha \cos \theta_{d}-\sin \alpha \sin \theta_{d},
\end{aligned}
$$

where $\theta_{d}$ is the projected angle corresponds to $\theta$ on the meridional plane. Using the above equations and Eq. (C.15) and taking that both $\theta$ and $\theta_{d}$ are small we find

$$
\begin{aligned}
\sin \theta_{d} & \approx \cos \phi \sin \theta \\
\text { or } \theta_{d} & \approx \theta \cos \phi .
\end{aligned}
$$

The colatitude with respect to magnetic axis

$$
\begin{aligned}
\theta & =\sin ^{-1}\left(\sqrt{r / r_{\mathrm{e}}}\right) \\
\text { or } \theta & \approx \sqrt{r / r_{\mathrm{e}}} .
\end{aligned}
$$

The rate of change of colatitude

$$
\begin{aligned}
\frac{\mathrm{d} \theta}{\mathrm{d} t} & =\frac{1}{2} \frac{1}{\sqrt{r\left(r_{\mathrm{e}}-r\right)}} \frac{\mathrm{d} r}{\mathrm{~d} t} \\
& \approx \frac{1}{2} \frac{1}{\sqrt{r r_{\mathrm{e}}}} \frac{\mathrm{d} r}{\mathrm{~d} t} .
\end{aligned}
$$

We also rewrite $\sin ^{2} \theta^{\prime}$ as

$$
\begin{aligned}
\sin ^{2} \theta^{\prime} & =\sin ^{2}\left(\alpha+\theta_{d}\right) \\
& =\frac{1}{2}\left[1-\cos (2 \alpha) \cos \left(2 \theta_{d}\right)+\sin (2 \alpha) \sin \left(2 \theta_{d}\right)\right] \\
& \approx \sin ^{2} \alpha\left[1+\theta_{d}^{2} \frac{\cos (2 \alpha)}{\left.\sin ^{2} \alpha\right]}\right. \\
& \approx \sin ^{2} \alpha\left[1-\frac{r}{r_{\mathrm{e}}} \cos ^{2} \phi\left(1-\cot ^{2} \alpha\right)\right] .
\end{aligned}
$$

\section{Appendix B: The expression form magnetic Lorentz force}

Since the field line tangent curves off from the direction of particle velocity $\left(\boldsymbol{v}_{\|}\right)$, an offset angle introduced between $\boldsymbol{v}_{\|}$and the field line tangent $\hat{b}$. This produces a non-vanishing magnetic Lorentz force $\boldsymbol{F}_{\mathrm{B}}$, which in tern constrains particle to the field line. The expression for $\boldsymbol{F}_{\mathrm{B}}$ in a $2 \mathrm{D}$ case has been derived by Gangadhara (1996). Here we are extending to find the relevant expression for $\boldsymbol{F}_{\mathrm{B}}$ in the 3D case.

The rotation velocity of a particle moving along a field line is given by

$\boldsymbol{v}_{\text {rot }}(r)=\boldsymbol{\Omega} \times \boldsymbol{r}=r \Omega \sin \theta^{\prime} \hat{\boldsymbol{\epsilon}}$,

where $\theta^{\prime}$ is the angle between rotation axis $\hat{\Omega}$ and the radial vector $\boldsymbol{r}$, and $\hat{\epsilon}$ is a unit vector in the direction of rotation velocity. If $\Theta$ is the angle between the field line tangent $\hat{b}$ and $\hat{\epsilon}$, then

$\hat{\epsilon}=\cos \Theta \hat{\epsilon}_{\|}+\sin \Theta \hat{\epsilon}_{\perp}$

where the unit vectors $\hat{\epsilon}_{\|}$and $\hat{\epsilon}_{\perp}$ are parallel and perpendicular to $\hat{b}$, respectively. The expression for $\Theta$ has been derived in G05.

The component of rotation velocity of particle perpendicular to the field line at a distance $r$ is given by

$\boldsymbol{v}_{\mathrm{p}}=v_{\mathrm{rot}}(r) \sin \Theta \hat{\epsilon}_{\perp}$, while the rotation velocity of field line at $r+\delta r$ is given by

$$
\boldsymbol{v}_{\mathrm{f}}=v_{\mathrm{rot}}(r+\delta r) \sin \Theta \hat{\epsilon}_{\perp}
$$

in the laboratory frame. Since the magnetic field is corotating with the pulsar different points on the field lines have different velocities for an observer in the laboratory frame. The particle at radial distance $r$ sees that the field line bends away from its velocity direction. Hence the relative velocity between the particle and the field line is given by

$\boldsymbol{v}_{\text {rel }}=\boldsymbol{v}_{\mathrm{p}}-\boldsymbol{v}_{\mathrm{f}}=\delta r \Omega \sin \theta^{\prime} \sin \Theta \hat{\epsilon}_{\perp}$,

where we have taken that both $\sin \Theta$ and $\sin \theta^{\prime}$ remain roughly constant over an incremental distance $\delta r$.

Since the gyration frequencies are very large compared to the radio wave frequencies the equation of motion Eq. (1) can be averaged over period of gyration particle (BCW91). Hence the effective magnetic Lorentz force is given by

$\boldsymbol{F}_{\mathrm{B}}=\frac{q}{c}\left(\boldsymbol{v}_{\mathrm{rel}} \times \boldsymbol{B}\right)$.

\section{Appendix C: The derivation of the equation of motion}

In the superstrong magnetic field of pulsar, charged particles lose their initial velocity component perpendicular to the magnetic field via synchrotron radiation. Hence the particle motion becomes effectively one dimensional along the magnetic field lines. We derive and solve the equation of motion of particles moving along the dipolar field lines.

\section{C.1. Particle motion along rotating magnetic field}

Consider a particle or a bunch moving along the magnetic field lines of an inclined and rotating dipole. For an observer in the co-rotating frame, the velocity of particle is given by

$\boldsymbol{v}_{\|}=\beta_{\|} c \hat{b}_{\mathrm{t}}$

where $\beta_{\|}$is a free parameter, reflecting the fraction of particle velocity along field line, $\hat{b}_{\mathrm{t}}=\boldsymbol{b}_{\mathrm{t}} /\left|\boldsymbol{b}_{\mathrm{t}}\right|$, and $\boldsymbol{b}_{\mathrm{t}}=\partial \boldsymbol{r} / \partial \theta$ is the tangent to magnetic field line of an inclined dipole (Gangadhara 2004, hereafter G04). The position vector of the particle is given by $r=r \hat{e}_{r}$, where $r=r_{\mathrm{e}} \sin ^{2} \theta, r_{\mathrm{e}}$ is the field line constant, $\theta$ the magnetic colatitude, and $\hat{e}_{r}$ the unit vector (see Eq. (C.5) below).

Next for an observer, who is at rest in the laboratory frame, the velocity of particle is given by

$\boldsymbol{v}_{\text {lab }}=\boldsymbol{v}_{\|}+\boldsymbol{\Omega} \times r$,

where $\boldsymbol{\Omega}$ is the pulsar angular velocity.

By differentiating

$r=r \hat{e}_{r}$

with respect to time in the laboratory frame, we obtain

$\boldsymbol{v}_{\mathrm{lab}}=\frac{\mathrm{d} r}{\mathrm{~d} t} \hat{e}_{r}+r \frac{\mathrm{d} \theta^{\prime}}{\mathrm{d} t} \hat{e}_{\theta}+r \sin \theta^{\prime} \frac{\mathrm{d} \phi_{p}}{\mathrm{~d} t} \hat{e}_{\phi}$,

where $\left(\hat{e}_{r}, \hat{e}_{\theta}, \hat{e}_{\phi}\right)$ are the basis vectors of spherical polar coordinate system centered on the rotation axis $\hat{\Omega}$. Since the equation of motion, i.e., Eq. (1) can be averaged over a gyration period of particle, the radial distance $r$ is effectively designating the guiding center location. 
The magnetic colatitude $\theta$ is measured in the coordinate frame with $z$ axis taken along magnetic axis $\hat{m}$ while the primed variable (colatitude) $\theta^{\prime}$ is measured in the coordinate frame with $z$ axis taken along $\hat{\Omega}$. The basis vectors and their derivatives with respect to time are given by

$$
\begin{aligned}
& \hat{e}_{r}=\sin \theta^{\prime}\left(\cos \phi_{\mathrm{p}} \hat{x}+\sin \phi_{\mathrm{p}} \hat{y}\right)+\cos \theta^{\prime} \hat{z}, \\
& \hat{e}_{\theta}=\cos \theta^{\prime}\left(\cos \phi_{\mathrm{p}} \hat{x}+\sin \phi_{\mathrm{p}} \hat{y}\right)-\sin \theta^{\prime} \hat{z}, \\
& \hat{e}_{\phi}=-\sin \phi_{\mathrm{p}} \hat{x}+\cos \phi_{\mathrm{p}} \hat{y},
\end{aligned}
$$

$\frac{\mathrm{d} \hat{e}_{r}}{\mathrm{~d} t}=\frac{\mathrm{d} \theta^{\prime}}{\mathrm{d} t} \hat{e}_{\theta}+\frac{\mathrm{d} \phi_{\mathrm{p}}}{\mathrm{d} t} \sin \theta^{\prime} \hat{e}_{\phi}$,

$\frac{\mathrm{d} \hat{e}_{\theta}}{\mathrm{d} t}=-\frac{\mathrm{d} \theta^{\prime}}{\mathrm{d} t} \hat{e}_{r}+\frac{\mathrm{d} \phi_{\mathrm{p}}}{\mathrm{d} t} \cos \theta^{\prime} \hat{e}_{\phi}$,

$\frac{\mathrm{d} \hat{e}_{\phi}}{\mathrm{d} t}=-\frac{\mathrm{d} \phi_{\mathrm{p}}}{\mathrm{d} t}\left(\sin \theta^{\prime} \hat{e}_{r}+\cos \theta^{\prime} \hat{e}_{\theta}\right)$,

\section{C.2. Particle phase}

The particle phase $\phi_{\mathrm{p}}$ with respect to a fixed co-ordinate system$\mathrm{XYZ}$ (see Fig. 1) is given by

$\phi_{\mathrm{p}}=\Omega t \pm \Delta \phi$,

where $\Delta \phi$ is the phase of particle measured with respect to the phase of magnetic axis $\hat{m}$ on the equatorial plane. The positive sign is for the leading side and negative for the trailing. The vector $\hat{m}$ can be defined as

$\hat{m}=\sin \alpha \cos (\Omega t) \hat{x}+\sin \alpha \sin (\Omega t) \hat{y}+\cos \alpha \hat{z}$.

If $\theta$ is the magnetic colatitude of particle then we have

$\hat{e}_{r} \cdot \hat{m}=\cos \theta$

$$
=\cos \alpha \cos \theta^{\prime}+\sin \alpha \sin \theta^{\prime} \cos (\Delta \phi) .
$$

By taking dot product of the vectors $\hat{\Omega}$ and $\hat{e}_{r}$, we obtain

$\hat{\Omega} \cdot \hat{e}_{r}=\cos \theta^{\prime}$,

and the expression for $\cos \theta^{\prime}$ is given recently in G05:

$\cos \theta^{\prime}=\cos \theta \cos \alpha-\sin \theta \sin \alpha \cos \phi$.

Using Eqs. (C.13) and (C.15), we find an expression for $\Delta \phi$ :

$\Delta \phi=\arccos \left(\frac{\cos \theta \sin \alpha+\cos \alpha \cos \phi \sin \theta}{\sin \theta^{\prime}}\right)$.

By taking the dot-product of $\hat{e}_{\phi}$ with Eqs. (C.2) and (C.4), and equating the right hand sides, we obtain

$r \sin \theta^{\prime} \frac{\mathrm{d} \phi_{\mathrm{p}}}{\mathrm{d} t}=(\boldsymbol{\Omega} \times r) \cdot \hat{e}_{\phi}+\frac{\mathrm{d} \theta}{\mathrm{d} t} \boldsymbol{b}_{\mathrm{t}} \cdot \hat{e}_{\phi}$.

Next, by simplify it, we obtain

$\Omega_{\mathrm{p}}=\Omega+\beta_{\|} c \frac{\cos \Theta}{r \sin \theta^{\prime}}$,

where $\Omega_{\mathrm{p}}=\mathrm{d} \phi_{\mathrm{p}} / \mathrm{d} t$ and $\cos \Theta=\hat{b}_{\mathrm{t}} \cdot \hat{e}_{\phi}$. The expression for $\cos \Theta$ is given in G05 (see Eq. (5)).

\section{C.3. Finding the equation of motion}

The momentum of particle is given by $\boldsymbol{p}_{\text {lab }}=m \boldsymbol{v}_{\mathrm{lab}}$, where $m=$ $m_{0} \gamma$, and the quantities $m$ and $m_{0}$ are the relativistic and the rest mass of particle, respectively. The Lorentz factor $\gamma$ is given by

$\gamma=\left(1-\frac{v_{\mathrm{lab}}^{2}}{c^{2}}\right)^{-1 / 2}$.

By substituting for $v_{\text {lab }}$ from Eq. (C.4), we obtain

$\gamma=\left[1-\frac{\dot{r}^{2}}{c^{2}}-\left(\frac{r}{c} \frac{\mathrm{d} \theta^{\prime}}{\mathrm{d} t}\right)^{2}-\left(\frac{r \sin \theta^{\prime} \Omega_{\mathrm{p}}}{c}\right)^{2}\right]^{-1 / 2}$,

where $\dot{r}$ is the time derivative of $\mathrm{r}$ with respect to time. Next, by substituting for $\boldsymbol{p}_{\text {lab }}$ into Eq. (1) and differentiating with respect to time, we obtain

$$
\begin{aligned}
& {\left[\frac{\mathrm{d}}{\mathrm{d} t}\left(m \frac{\mathrm{d} r}{\mathrm{~d} t}\right)-m r\left(\frac{\mathrm{d} \theta^{\prime}}{\mathrm{d} t}\right)^{2}-m r \Omega_{\mathrm{p}}^{2} \sin ^{2} \theta^{\prime}\right] \hat{e}_{r} } \\
+ & {\left[\frac{\mathrm{d}}{\mathrm{d} t}\left(m r \frac{\mathrm{d} \theta^{\prime}}{\mathrm{d} t}\right)+m \frac{\mathrm{d} r}{\mathrm{~d} t} \frac{\mathrm{d} \theta^{\prime}}{\mathrm{d} t}-m r \Omega_{\mathrm{p}}^{2} \sin \theta^{\prime} \cos \theta^{\prime}\right] \hat{e}_{\theta} } \\
+ & {\left[m \Omega_{\mathrm{p}} \frac{\mathrm{d}}{\mathrm{d} t}\left(r \sin \theta^{\prime}\right)+\frac{\mathrm{d}}{\mathrm{d} t}\left(m r \Omega_{\mathrm{p}} \sin \theta^{\prime}\right)\right] \hat{e}_{\phi} } \\
= & q \boldsymbol{E}+\boldsymbol{F}_{\mathrm{B}} .
\end{aligned}
$$

For the convenience of understanding the motion, we resolve the equation into radial and non-radial parts, i.e., along $\hat{e}_{r}$ and in the plane: $\left(\hat{e}_{\theta}, \hat{e}_{\phi}\right)$. Hence, by separating the terms of Eq. (C.21) into radial and non-radial parts, we obtain

$\left[\frac{\mathrm{d}}{\mathrm{d} t}\left(m \frac{\mathrm{d} r}{\mathrm{~d} t}\right)-m r\left(\frac{\mathrm{d} \theta^{\prime}}{\mathrm{d} t}\right)^{2}\right] \hat{e}_{r}=\boldsymbol{F}_{\mathrm{B} r}+\left(q \boldsymbol{E}_{r}+\boldsymbol{F}_{\mathrm{c} r}\right)$

and

$\boldsymbol{F}_{\mathrm{U}}=\boldsymbol{F}_{\mathrm{B} \theta \phi}+q \boldsymbol{E}_{\theta \phi}$,

where $\boldsymbol{F}_{\mathrm{B} r}$ and $\boldsymbol{F}_{\mathrm{B} \theta \phi}=\boldsymbol{F}_{\mathrm{B} \theta}+\boldsymbol{F}_{\mathrm{B} \phi}$ are the radial and non-radial components of $\boldsymbol{F}_{\mathrm{B}}$, respectively. Similarly, $\boldsymbol{E}_{r}$ and $\boldsymbol{E}_{\theta \phi}=\boldsymbol{E}_{\theta}+\boldsymbol{E}_{\phi}$ are the components of $\boldsymbol{E}$. The quantities $\boldsymbol{F}_{\mathrm{B} r}, \boldsymbol{F}_{\mathrm{B} \theta}$ and $\boldsymbol{F}_{\mathrm{B} \phi}$ are the components of $\boldsymbol{F}_{\mathrm{B}}$ in the directions of $\left(\hat{e}_{r}, \hat{e}_{\theta}, \hat{e}_{\phi}\right)$, respectively. Similarly, $\boldsymbol{E}$ has the components in these directions.

The centrifugal force is given by

$$
\begin{aligned}
\boldsymbol{F}_{\mathrm{c}} & =m \Omega_{\mathrm{p}}^{2} \hat{\Omega} \times(\boldsymbol{r} \times \hat{\Omega}) \\
& =m r \sin \theta^{\prime} \Omega_{\mathrm{p}}^{2} \hat{e}_{\mathrm{p}},
\end{aligned}
$$

where

$\hat{e}_{\mathrm{p}}=\sin \theta^{\prime} \hat{e}_{r}+\cos \theta^{\prime} \hat{e}_{\theta}=\cos \phi_{\mathrm{p}} \hat{x}+\sin \phi_{\mathrm{p}} \hat{y}$.

Then the component of centrifugal force in the $\hat{e}_{r}$ direction is given by

$\boldsymbol{F}_{\mathrm{c} r}=\left(\boldsymbol{F}_{\mathrm{c}} \cdot \hat{e}_{r}\right) \hat{e}_{r}$

The quantity $\boldsymbol{F}_{\mathrm{U}}$ is the sum of the Coriolis force, the force generated due to non-uniform rotation velocity $\boldsymbol{v}_{\text {rot }}$ and the component of centrifugal force in the $\hat{e}_{\theta}$ and $\hat{e}_{\phi}$ directions:

$$
\begin{aligned}
\boldsymbol{F}_{\mathbf{U}} & =\left[\frac{\mathrm{d}}{\mathrm{d} t}\left(m r \frac{\mathrm{d} \theta^{\prime}}{\mathrm{d} t}\right)+m \frac{\mathrm{d} r}{\mathrm{~d} t} \frac{\mathrm{d} \theta^{\prime}}{\mathrm{d} t}-m r \Omega_{p}^{2} \sin \theta^{\prime} \cos \theta^{\prime}\right] \hat{e}_{\theta} \\
& +\left[m \Omega_{p} \frac{\mathrm{d}}{\mathrm{d} t}\left(r \sin \theta^{\prime}\right)+\frac{\mathrm{d}}{\mathrm{d} t}\left(m r \sin \theta^{\prime} \Omega_{p}\right)\right] \hat{e}_{\phi} .
\end{aligned}
$$


Since the detailed study of particle motion is beyond the scope of this work, we intend to qualitatively understand the constrained motion. The following deductions are based on the presumption that the charged particles closely follow the field lines over the range of radial distance of our interest, i.e., the radio emission region as mentioned earlier.

Initially, particles can have a velocity component $\left(\boldsymbol{v}_{\perp}\right)$ perpendicular to the magnetic field. In the superstrong magnetic field of pulsar, particles lose their perpendicular component of motion via synchrotron radiation losses. Therefore, they essentially have one dimensional motion along the field lines. Since particles move along rotating field lines, there will be a relative non-zero rotation velocity $\boldsymbol{v}_{\text {rel }}$ between the particles and the field lines. Hence particles try to gyrate due to the action of magnetic Lorentz force: $\boldsymbol{F}_{\mathrm{B}}=q / c\left(\boldsymbol{v}_{\text {rel }} \times \boldsymbol{B}\right)$, but the field lines curves off to the side. The motion of particles in curved magnetic field lines is discussed by Jackson (1975). The expression for $\boldsymbol{v}_{\text {rel }}$ is derived in Appendix B. The forces $\boldsymbol{F}_{\mathrm{U}}, \boldsymbol{F}_{\mathrm{B} \theta \phi}$ and $q \boldsymbol{E}_{\theta \phi}$ act as constraining forces. They balance in such a away that the particle is hardly allowed to deviate away from the field line. This bead-on-wire approximation holds till the aforementioned force balance is maintained. The bead-on-wire approximation is expected to fail near the light cylinder, as the inertial forces such as Coriolis force exceeds the Lorentz force. Because the rotation speed approaches the speed of light and magnetic dipole field becomes weaker near the light cylinder.

If $\theta_{\mathrm{B}}$ is the angle between $\hat{e}_{\mathrm{r}}$ and $\hat{b}$, then we have

$$
\begin{aligned}
\sin \theta_{\mathrm{B}} & =\sqrt{\left(\hat{e}_{r} \times \hat{b}_{t}\right) \cdot\left(\hat{e}_{r} \times \hat{b}_{t}\right)} \\
& =\frac{\sqrt{2} \sin \theta}{\sqrt{5+3 \cos (2 \theta)}} .
\end{aligned}
$$

We find $\sin \theta_{\mathrm{B}} \ll 1$ within the region of $10 \% r_{\mathrm{L}}$ in the case of normal pulsars, but $30 \% r_{\mathrm{L}}$ in millisecond pulsars, therefore, we infer that $\left|\boldsymbol{F}_{\mathrm{Br}}\right| \ll\left|\boldsymbol{F}_{\mathrm{B} \theta \phi}\right|$.

By substituting for $\boldsymbol{F}_{\mathrm{c} r}$ into Eq. (C.22), we obtain the equation of motion in radial direction:

$$
\begin{aligned}
& {\left[\frac{\mathrm{d}}{\mathrm{d} t}\left(m \frac{\mathrm{d} r}{\mathrm{~d} t}\right)-m r\left(\frac{\mathrm{d} \theta^{\prime}}{\mathrm{d} t}\right)^{2}-m r \sin ^{2} \theta^{\prime} \Omega_{\mathrm{p}}^{2}\right] \hat{e}_{r}} \\
& =q \boldsymbol{E}_{r}+\boldsymbol{F}_{\mathrm{B} r} .
\end{aligned}
$$

The component $\boldsymbol{E}_{r} \sim \boldsymbol{E}_{\|}$is negligible as the component of electric field parallel to the field lines is screened by the $\left(\mathrm{e}^{+}, \mathrm{e}^{-}\right)$ plasma. Since $\theta_{\mathrm{B}} \sim 0^{\circ}$, the force term $\boldsymbol{F}_{\mathrm{B} r}$ becomes negligibly small compared to the other terms in Eq. (C.27). Hence the equation of motion in the radial direction reduces to

$$
\frac{\mathrm{d}}{\mathrm{d} t}\left(m \frac{\mathrm{d} r}{\mathrm{~d} t}\right)-m r\left(\frac{\mathrm{d} \theta^{\prime}}{\mathrm{d} t}\right)^{2}-m r \sin ^{2} \theta^{\prime} \Omega_{\mathrm{p}}^{2}=0 .
$$

In the limiting case of $\theta=0^{\circ}$ and $\mathrm{d} \theta / \mathrm{d} t=0$, i.e., for a particle following the magnetic axis of an orthogonal rotator $\left(\alpha=90^{\circ}\right)$, we can show that the Eq. (C.28) reduces to the 2D equation of motion derived by Gangadhara (1996).

\section{Appendix D: The solution of Eq. (C.28)}

Here we develop a perturbative method for solving Eq. (6). We consider $\epsilon=r_{\mathrm{L}} / r_{\mathrm{e}}$ as perturbative parameter.

The series expansions of $r$ and $\dot{r}$ in terms of $\epsilon$ are

$r=r_{0}+\epsilon r_{1}+\epsilon^{2} r_{2}+\cdots$, and

$\dot{r}=\dot{r}_{0}+\epsilon \dot{r}_{1}+\epsilon^{2} \dot{r}_{2}+\cdots$

Thus by substituting Eqs. (D.1) and (D.2) into Eq. (7), and series expanding it we obtain

$\Omega_{\mathrm{m}}^{2}=\Omega_{\mathrm{m} 0}^{2}+\epsilon \Omega_{\mathrm{m} 1}^{2}+\epsilon^{2} \Omega_{\mathrm{m} 2}^{2}+\cdots$

and

$\frac{\mathrm{d} \Omega_{\mathrm{m}}^{2}}{\mathrm{~d} t}=\dot{\Omega_{\mathrm{m}}^{2}}=\Omega_{\mathrm{m} 0}^{\dot{2}}+\epsilon \Omega_{\mathrm{m} 1}^{\dot{2}}+\epsilon^{2} \Omega_{\mathrm{m} 2}^{\dot{2}}+\cdots$

where the perturbation expansion coefficients, which follow from Eqs. (7), (A.1) and (A.6), are

$\Omega_{\mathrm{m} 0}^{2}=\Omega^{2} \sin ^{2} \alpha$,

$\Omega_{\mathrm{m} 1}^{2}=R_{1} \frac{\dot{r}_{0}^{2}}{r_{0} r_{\mathrm{L}}}+R_{2} \frac{r_{0}}{r_{\mathrm{L}}}$,

$\Omega_{\mathrm{m} 0}^{\dot{2}}=0$

$\Omega_{\mathrm{m} 1}^{\dot{2}}=R_{1}\left(\frac{2 \dot{r_{0}} \ddot{r}_{0}}{r_{0} r_{\mathrm{L}}}-\frac{\dot{r}_{0}^{3}}{r_{0}^{2} r_{\mathrm{L}}}\right)+R_{2} \frac{\dot{r}_{0}}{r_{\mathrm{L}}}$,

and

$R_{1}=\frac{1}{8}[3-\cos (2 \phi)]$,

$R_{2}=\frac{\Omega^{2}}{4}\left[1+3 \cos (2 \alpha)-2 \cos (2 \phi) \sin ^{2} \alpha\right]$.

After substituting Eqs. (D.1)-(D.6) into Eq. (6), the coefficients of $\epsilon^{0}$ give the zeroth order equation:

$\ddot{r}_{0}+\frac{2 \Omega_{\mathrm{m} 0}^{2} r_{0}{\dot{r_{0}}}^{2}}{c^{2}\left(1-\Omega_{\mathrm{m} 0}^{2} r_{0}^{2} / c^{2}\right)}-\Omega_{\mathrm{m} 0}^{2} r_{0}=0$,

and those of $\epsilon$ give the first order equation:

$$
\begin{gathered}
\ddot{r}_{1}+\frac{4 \Omega_{\mathrm{m} 0}^{2} r_{0} \dot{r}_{0}}{c^{2}\left(1-\Omega_{\mathrm{m} 0}^{2} r_{0}^{2} / c^{2}\right)} \dot{r}_{1}+\left(\frac{2 \Omega_{\mathrm{m} 0}^{2} \dot{r}_{0}^{2}}{c^{2}\left(1-\Omega_{\mathrm{m} 0}^{2} r_{0}^{2} / c^{2}\right)}\right. \\
\left.-\Omega_{\mathrm{m} 0}^{2}+\frac{4 \Omega_{\mathrm{m} 0}^{4} r_{0}^{2} \dot{r}_{0}^{2}}{c^{4}\left(1-\Omega_{\mathrm{m} 0}^{2} r_{0}^{2} / c^{2}\right)^{2}}\right) r_{1}= \\
\Omega_{\mathrm{m} 1}^{2} r_{0}-\frac{2 \Omega_{\mathrm{m} 1}^{2} r_{0} \dot{r}_{0}^{2} \Omega_{\mathrm{m} 1}^{2} \dot{r}_{0} r_{0}^{2}}{c^{2}\left(1-\Omega_{\mathrm{m} 0}^{2} r_{0}^{2} / c^{2}\right)} \\
-\frac{2 \Omega_{\mathrm{m} 0}^{2} \Omega_{\mathrm{m} 1}^{2} \dot{r}_{0}^{2} r_{0}^{3}}{c^{4}\left(1-\Omega_{\mathrm{m} 0}^{2} r_{0}^{2} / c^{2}\right)^{2}} .
\end{gathered}
$$

\section{D.1. The solution}

We employed the method, which has been used in our 2D model (TG05), to find the analytical solution to zeroth order equation. Thus the solution of the zeroth order equation is given by

$r_{0}=\frac{c}{\Omega_{\mathrm{m} 0}} \operatorname{cn}\left(\lambda-\Omega_{\mathrm{m} 0} \mathrm{t}\right)$,

where $\operatorname{cn}(z)$ is the Jacobian Elliptical cosine function (Abramowicz \& Stegun 1972) and

$\lambda=\int_{0}^{\phi_{0}} \frac{\mathrm{d} \zeta}{\sqrt{1-k^{2} \sin ^{2} \zeta}}$ 
The functions $k$ and $\phi_{0}$ are given by

$k^{2}=\frac{1}{1-s_{0}^{2}}\left[1-\frac{{\dot{s_{0}}}^{2}}{\left(1-s_{0}^{2}\right) \Omega^{2}}\right]$,

and

$\phi_{0}=\arccos \left(\frac{r_{\mathrm{i}} \Omega_{\mathrm{m} 0}}{c}\right)$,

where $s_{0}=\left.s\right|_{r_{0}=r_{\mathrm{i}}}, \dot{s}_{0}=\mathrm{d} s /\left.\mathrm{d} t\right|_{t=0}$, and $s=\Omega_{\mathrm{m} 0} r_{0} / c$. The parameter $r_{\mathrm{i}} \sim 10^{6} \mathrm{~cm}$ is the initial radial location of particle at time $t=0$. The expression given by Eq. (D.9) is an exact solution for particles moving on the magnetic axis, and it is applicable to both the normal and the millisecond pulsars. We substitute the approximate form of Eq. (D.9) into Eq. (D.8) to find a solution to the first order equation. Since $k \approx 0$, we can approximate $r_{0}$ and obtain

$r_{0} \approx \frac{c}{\Omega_{\mathrm{m} 0}} \sin \left(\Omega_{\mathrm{m} 0} \mathrm{t}\right)$.

We seek for a solution for the first order equation which is valid over a distance $10 \% r_{\mathrm{L}}$ from the neutron star surface. Since $\Omega_{\mathrm{m}} t \ll 1$ over this range of altitude, we can take $\sin \left(\Omega_{\mathrm{m} 0} t\right) \approx$ $\Omega_{\mathrm{m} 0} t$ and

$\left(\frac{\dot{r}_{0}}{c}\right)^{2}=1-\frac{r_{0}^{2} \Omega_{\mathrm{m} 0}^{2}}{c^{2}} \approx 1$.

Using the approximations (D.12) and (D.13) we can rewrite Eq. (D.8):

$$
\begin{aligned}
\ddot{r}_{1}+ & 4 Q_{1} t \dot{r}_{1}+Q_{1}\left(1+4 Q_{1} t^{2}\right) r_{1}= \\
& P_{2} t^{2}+P_{4} t^{4},
\end{aligned}
$$

where

$Q_{1}=\Omega_{\mathrm{m} 0}^{2}$,

$P_{2}=-\frac{2 c^{2}\left(R_{1} \Omega_{\mathrm{m} 0}^{2}+R_{2}\right)}{r_{\mathrm{L}}}$,

$P_{4}=-\frac{2 c^{2} R_{2} \Omega_{\mathrm{m} 0}^{2}}{r_{\mathrm{L}}}$.

The homogeneous part of Eq. (D.14) is given by

$\ddot{h}+4 Q_{1} t \dot{h}+Q_{1}\left(1+4 Q_{1} t^{2}\right) h=0$,

where $h$ is the contribution to $r_{1}$ from homogeneous part. By defining

$h=h_{0}(t) \exp \left[-Q_{1} t^{2}\right]$

we can reduce Eq. (D.16):

$\ddot{h_{0}}=Q_{1} h_{0}$.

The solution of Eq. (D.18) is given by

$h_{0}=C_{1} \sinh \left(\sqrt{Q_{1}} t\right)+C_{2} \cosh \left(-\sqrt{Q_{1}} t\right)$,

where $C_{1}$ and $C_{2}$ are arbitrary constants. We take the initial condition that $h_{0}=0$ and $\dot{h_{0}}=v_{p 0}$ and $v_{p 0} \ll c$ at time $t=0$. Thus we have $C_{1}=v_{p 0} / \sqrt{Q_{1}}$ and $C_{2}=0$, and the solution $h_{0}$ can be written

$h_{0}=\frac{C_{1}}{2}\left(\exp \left[\sqrt{Q_{1}} t\right]-\exp \left[-\sqrt{Q_{1}} t\right]\right]$ where Since $\exp \left[\sqrt{Q_{1}} t\right]$ and $\exp \left[-\sqrt{Q_{1}} t\right]$ independently satisfy Eq. (D.18), we can write the solution for $r_{1}$ as

$r_{1}=-y_{1} \int \frac{y_{2} \kappa}{w} \mathrm{~d} t+y_{2} \int \frac{y_{1} \kappa}{w} \mathrm{~d} t$

where

$y_{1}=\exp \left[-Q_{1} t^{2}+\sqrt{Q_{1}} t\right]$,

$y_{2}=-\exp \left[-Q_{1} t^{2}-\sqrt{Q_{1}} t\right]$,

$w=y_{2} \frac{\mathrm{d} y_{1}}{\mathrm{~d} t}-y_{1} \frac{\mathrm{d} y_{2}}{\mathrm{~d} t}$

is the Wronskian (e.g., Zwillinger 1989), and $\kappa=P_{2} t^{2}+P_{4} t^{4}$. We numerically evaluated the integrals in Eq. (D.20) using Mathematica.

\section{Appendix E: The relation between $\rho_{\text {dipole }}$ and $\rho_{\text {rot }}$}

By finding the derivative of $v_{\mathrm{lab}}$, we can write acceleration as:

$\boldsymbol{a}=\frac{\mathrm{d} v_{\mathrm{lab}}}{\mathrm{d} t}$

Hence, following Eq. (13), we find

$\rho_{\text {rot }}=\frac{\left|\boldsymbol{v}_{\mathrm{lab}}\right|^{2}}{|\boldsymbol{a}|}$

where

$\left|\boldsymbol{v}_{\text {lab }}\right|^{2}=v_{0}^{2}+v_{\text {rot }}^{2}$

and

$v_{0}^{2}=\frac{\mathrm{d} r^{2}}{\mathrm{~d} t}+\left(r \frac{\mathrm{d} \theta^{\prime}}{\mathrm{d} t}\right)^{2}+\left(r \sin \theta^{\prime} F\right)^{2}$

$v_{\mathrm{rot}}^{2}=\left(r \sin \theta^{\prime}\right)^{2}\left(\Omega^{2}+2 \Omega F\right)$

where $F=\beta_{\|} c \cos \Theta / r \sin \theta^{\prime}$. The expression for $\boldsymbol{a}$ can be expressed as

$\boldsymbol{a}=a_{r} \hat{e}_{r}+a_{\theta} \hat{e}_{\theta}+a_{\phi} \hat{e}_{\phi}$.

We can split the components as

$a_{r}=a_{r 0}+a_{r r}$,

$a_{\theta}=a_{\theta 0}+a_{\theta r}$,

$a_{\phi}=a_{\phi 0}+a_{\phi r}$,

where

$a_{r 0}=\frac{\mathrm{d}}{\mathrm{d} t}\left(\frac{\mathrm{d} r}{\mathrm{~d} t}\right)-r\left(\frac{\mathrm{d} \theta^{\prime}}{\mathrm{d} t}\right)^{2}-r \sin ^{2} \theta^{\prime} F^{2}$

$a_{\theta 0}=\frac{\mathrm{d}}{\mathrm{d} t}\left(r \frac{\mathrm{d} \theta^{\prime}}{\mathrm{d} t}\right)+\frac{\mathrm{d} r}{\mathrm{~d} t} \frac{\mathrm{d} \theta^{\prime}}{\mathrm{d} t}-r F^{2} \sin \theta^{\prime} \cos \theta^{\prime}$

$a_{\phi 0}=F \frac{\mathrm{d}}{\mathrm{d} t}\left(r \sin \theta^{\prime}\right)+$

$\frac{\mathrm{d}}{\mathrm{d} t}\left(r F \sin \theta^{\prime}\right)$

and

$a_{r r}=-r \sin ^{2} \theta^{\prime}\left(\Omega^{2}+2 \Omega F\right)$

$a_{\theta r}=-r \sin \theta^{\prime} \cos \theta^{\prime}\left(\Omega^{2}+2 \Omega F\right)$

$a_{\phi r}=2 \Omega \frac{\mathrm{d}}{\mathrm{d} t}\left(r \sin \theta^{\prime}\right)$. 
We write the term $a=\sqrt{a_{r}^{2}+a_{\theta}^{2}+a_{\phi}^{2}}=\sqrt{a_{0}^{2}+a_{\mathrm{rot}}^{2}}$ and

$a_{0}^{2}=a_{r 0}^{2}+a_{\theta 0}^{2}+a_{\phi 0}^{2}$

$a_{\mathrm{rot}}^{2}=2\left(a_{r 0} a_{r r}+a_{\phi 0} a_{\phi r}+a_{\theta 0} a_{\theta r}\right)+a_{r r}^{2}+a_{\theta r}^{2}+a_{\phi r}^{2}$.

In the absence of rotation, the radius of curvature of particle trajectory becomes same as the radius of curvature of field line along which it is constrained to move. We can also find that the terms $v_{\mathrm{rot}}^{2}$ and $a_{\mathrm{rot}}^{2}$ vanish in the absence of rotation because of the $\Omega$ term. Thus we can write

$\rho_{\text {dipole }}=\frac{v_{0}^{2}}{a_{0}}$.

After a few algebraic manipulations with the above said terms and using Eq. (E.2) we can write the relation between $\rho_{\text {rot }}$ and $\rho_{\text {dipole }}$ as

$\rho_{\text {rot }}=\rho_{\text {dipole }}\left(\frac{1+v_{\mathrm{rot}}^{2} / v_{0}^{2}}{\sqrt{1+a_{\mathrm{rot}}^{2} / a_{0}^{2}}}\right)$. 